Hussein S., Jassim S., Al-Assam H. (2017) Automatic Quantification of Epidermis Curvature in H\&E Stained Microscopic Skin Image of Mice. In: Valdés Hernández M., González-Castro V. (eds) Medical Image Understanding and Analysis. MIUA 2017. Communications in Computer and Information Science, vol 723. Springer, Cham

The final publication is available at Springer via https://doi.org/10.1007/978-3-319-60964$5 \_81$ 


\title{
Automatic Quantification of Epidermis Curvature in H\&E Stained Microscopic Skin Image of Mice
}

\author{
Saif Hussein, Sabah Jassim, Hisham Al-Assam \\ Applied Computing Department, The University of Buckingham, Buckingham, UK
}

\{fistnam.lastname@buckingham.ac.uk\}

\begin{abstract}
Changes in the curvature of the epidermis layer is often associated with many skin disorders, such as ichthyoses and generic effects of ageing. Therefore, methods to quantify changes in the curvature are of a scientific and clinical interest. Manual methods to determine curvature are both laborious and intractable to large scale investigations. This paper proposes an automatic algorithm to quantify curvature of microscope images of H\&E-stained murine skin. The algorithm can be divided into three key stages. First, skin layers segmentation based on colour deconvolution to separate the original image into three channels of different representations to facilitate segmenting the image into multiple layers, namely epidermis, dermis and subcutaneous layers. The algorithm then further segments the epidermis layer into cornified and basal sub-layers. Secondly, it quantifies the curvature of the epidermis layer by measuring the difference between the epidermis edge and a straight line (theoretical reference line) connecting the two far sides of the epidermis edge. Finally, the curvature measurements extracted from a large number of images of mutant mice are used to identify a list of genes responsible for changes in the epidermis curvature. A dataset of $5714 \mathrm{H} \& \mathrm{E}$ microscopic images of mutant and wild type mice were used to evaluate the effectiveness of the algorithm.
\end{abstract}

Keywords: skin layer segmentation, epidermes layer quantification, skin curvature quantification.

\section{Introduction}

Although the Human Genome Project (HGP) determined the sequence of chemical base pairs, which make up human DNA and of identifying and mapping all of the genes of the human genome (approximately 20,500 genes), functionalities of the vast majority of genes are still under research or unknown [1]. The Mouse Genetics Project (MGP) is a large-scale mutant mouse production and phenotyping program aimed at identifying new model organisms of disease. The aim of the MGP is to produce over 20,000 mutant lines and the results are then translated into diagnostics, and treatments for diseases.

Mammalian skin is a complex organ composed of a variety of cell and tissue types. It is the largest mammalian organ, and although apparently simple, it is a highly organized tissue comprised of the epidermis, underlying dermis containing connective tissue and a deeper subcutaneous adipose layer. [2].The skin can reveal evidence of inflammation, hyperplasia, connective tissue disorders and underlying metabolic 
changes resulting from local and systemic influences. As dermatology research requires a detailed understanding of skin structure and organization, which requires quantitative measurements, the automatic quantification of changes in skin structures has a variety of different applications for biological research. One requirement in this area is the accurate segmentation of the skin compartments followed by the assessment of various characteristics of those compartments. For example, changes in the curvature of the epidermis layer are often associated with many skin disorders, such as ichthyoses and generic effects of ageing [3]. Therefore, methods to quantify changes in the curvature are of a scientific and clinical interest. Arguably, this cannot be achieved without a reliable segmentation of different skin layers.

Traditional skin studies have utilized manual methods for the quantification of skin features, which is a challenging task due to the complexity of the analysis with big amount of datasets. However, there has been recently a move towards the automation of these techniques to improve accuracy and efficiency by reducing processing time and laboratory costs.

In previous works, one of the attempts to automate the analysis of skin layer in microscopic images was the introduction of the novel shapelet-based procedure for the epidermis boundaries identification and thickness measurement [4]. Later, a classification method was proposed to segment skin layers in images based only on their speckle information [5]. Another interesting work was a hybrid sequence segmentation and classification technique applied to split images into different channels by zstack deconvolution, fitting model of skin layer and their classification into epidermis and dermis [6]. Recently high-definition optical coherence tomography was employed for grey imaging subsurface skin tissues to segment the epidermis layer with using weighted least square based edge-preserving smoothing method with weighted median filter followed by wavelet techniques an [7]

Although all those methods gave great results, they do not allow automatic quantification of epidermis curvature, which is a crucial feature for gene identification and skin disorders diagnostic. In addition to above there wasn't any of methods achieve sub-segmentation of the epidermis layer into cornified and basal layer

In this paper, we propose an automated method to quantify and measure the curvature of the epidermis layer (cornified sub-layer). The ultimate aim is to use the measurements extracted from a large number of mutant mice to identify a list of genes responsible for significant alterations in the curvature of the epidermis.

The rest of the paper is organised as follows. Section 2 explains the proposed algorithm in details whereas section 3 presents the method of identifying the interesting genes using reference range (RR). Section 4 presents the data set used to test the proposal algorithm as well as the result of interesting genes responsible for changes in the epidermis curvature. Finally the section 5 summarises the whole paper by clarifying the aims of this work. 


\section{The proposed Method}

The proposed method consist of an algorithm that automatically computes the curvature along the epidermis layer and identify the genes associated with significant changes in the curvature. It is summarized with an illustration in Figure 1, and works in 3 key stages.

1. Segments the skin image into its three layers, namely Epidermis, Dermis and Subcutaneous layers. And further segments the Epidermis layer into Cornified and Basal sub-layers [8].

2. Quantify the curvature of the epidermis layer by measuring the difference between the epidermis edge and a straight line (an artificial reference line) connecting the two far sides of the epidermis edge.

3. Using the curvature measurements extracted from a large number of skin images of mutant mice to identify a list of genes responsible for changes in the epidermis curvature.

The following subsections explain each of the key stages in details.

Fig. 1. The proposed algorithm to quantify the curvature of the Epidermis layer to identify the genes associated with significant changes in it

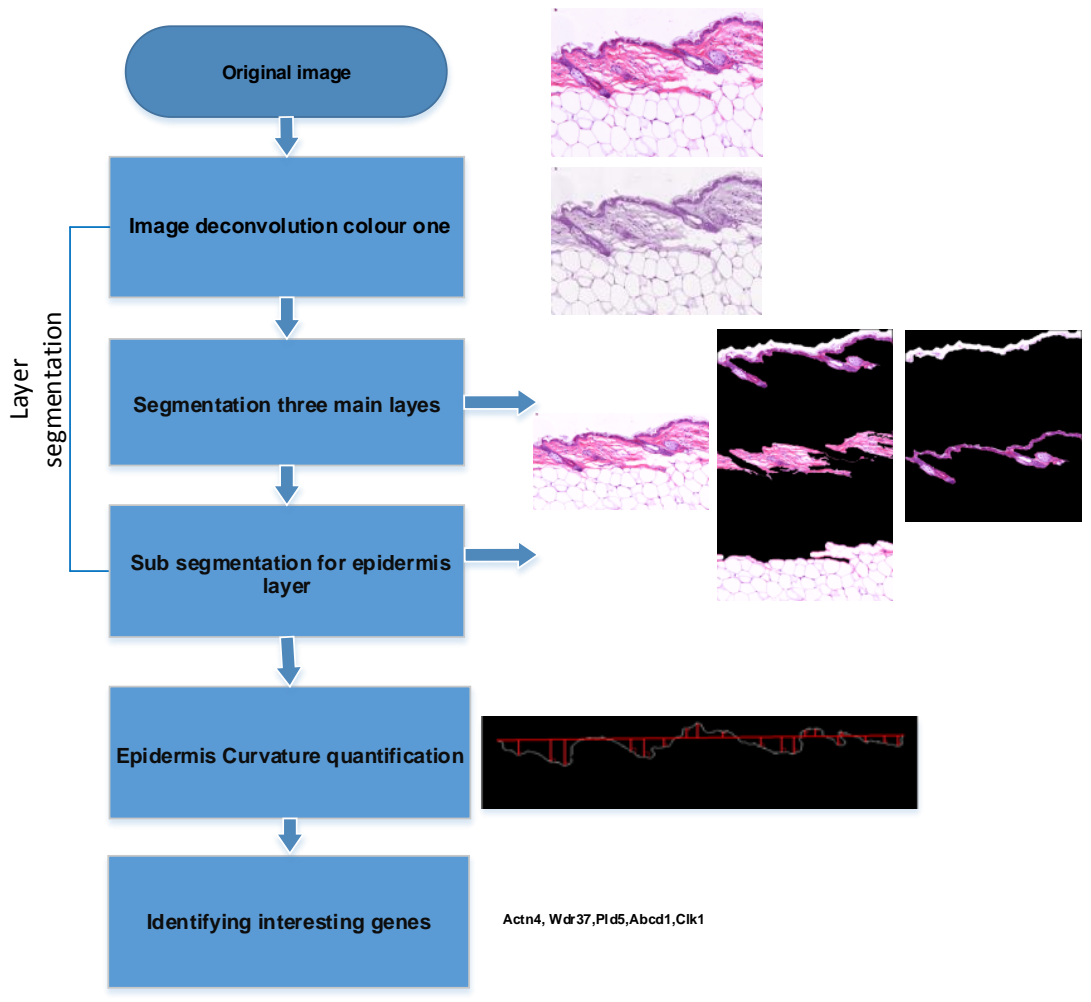




\subsection{Layers Segmentation algorithm}

Segmentation is an important step in the algorithm because the quality of the results is impacted by the quality of the underlying segmentation. Our algorithm uses adaptive colour deconvolution techniques on the H\&E stain images to separate different tissue structures as shown in Figure 2 [8]. Then the algorithm uses a set of morphological operations with appropriate structural elements, such as image open, image close and image fill a gap. To further remove unwanted objects, logical operations, such as adding and multiplying images, have been applied to segment epidermis sub layers as illustrated in Figure 3.

Fig. 2.Colour deconvolution A) Original image. B) Colour 2 deconvolution. C) Colour 3 deconvolution.
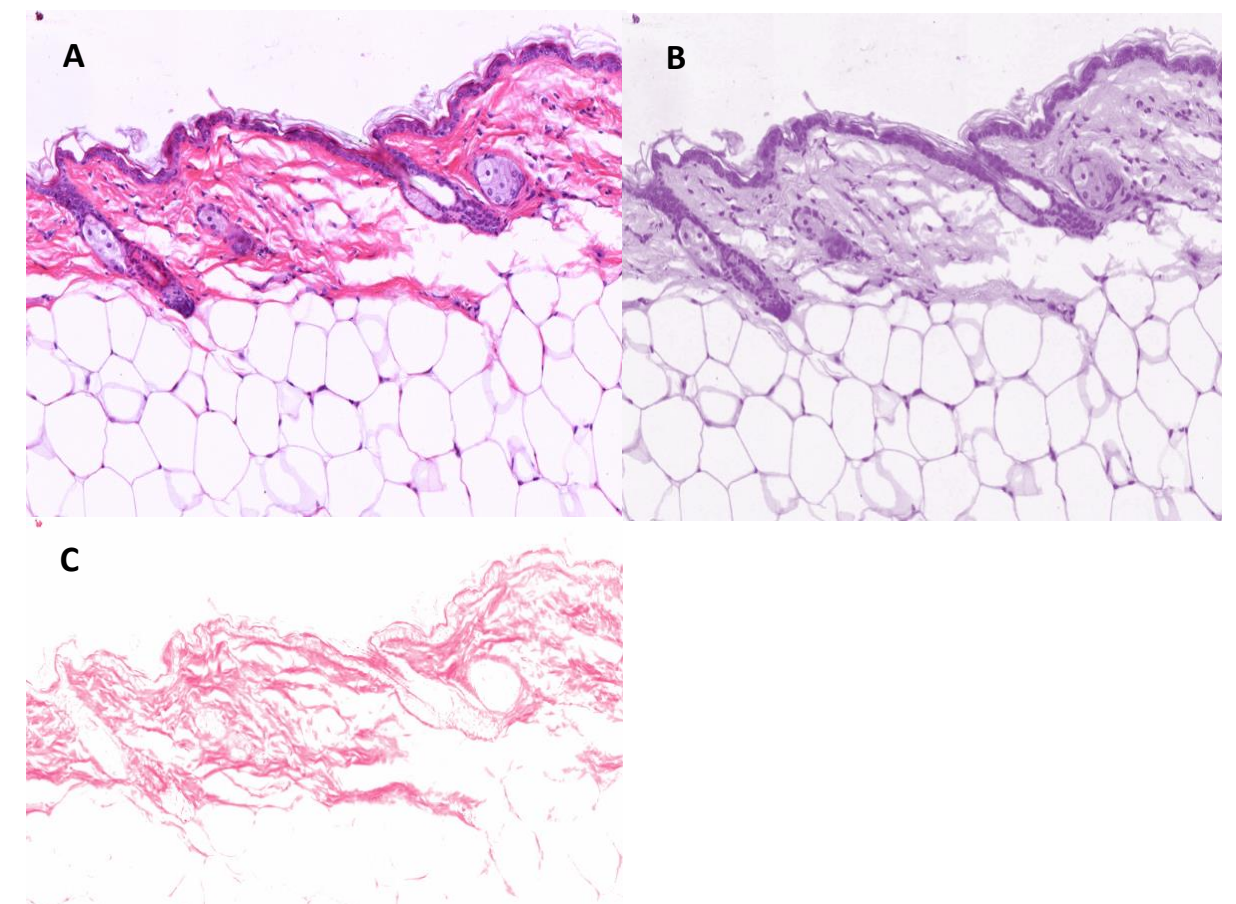
Fig. 3. Automatic method for layers and sub-layers segmentation A) Original image. B) Epidermis layer segmentation. C) Dermis layer segmentation. D) Sub-cutaneous layer segmentation. E) Cornified sub layer segmentation. F) Basal sub layer segmentation.

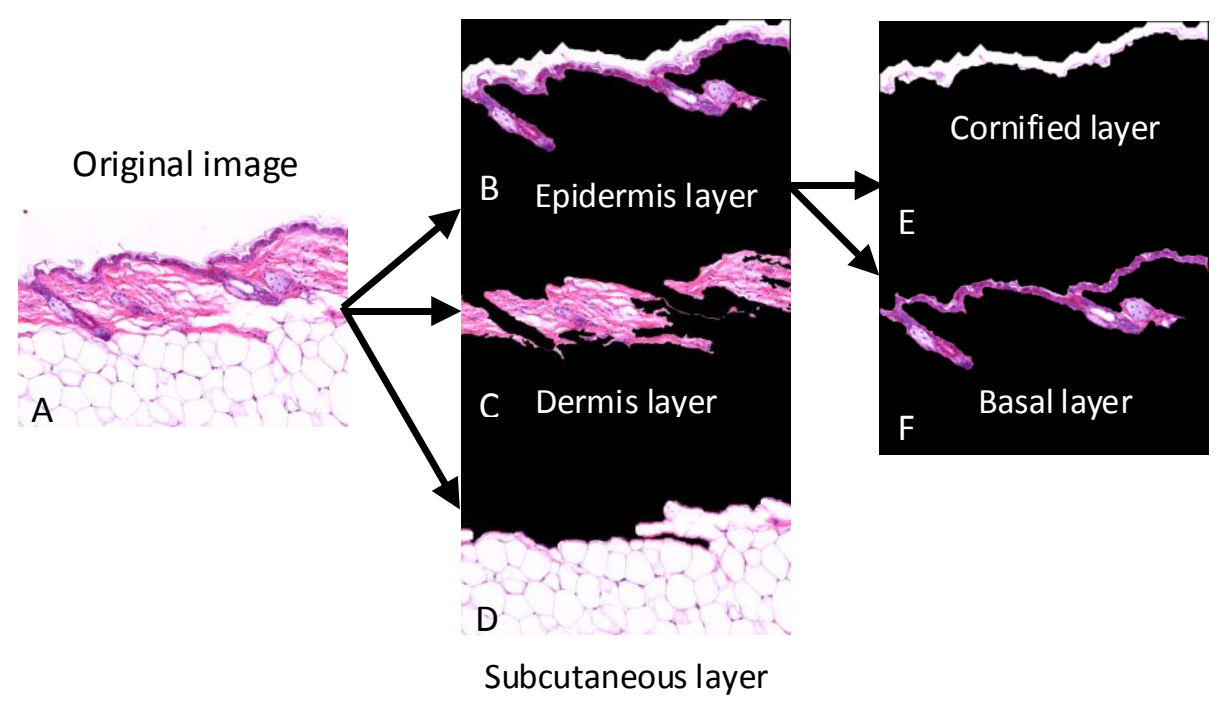

\subsection{Quantifying the Curvature of the Epidermis Layer}

Accurate quantification of the curvature of the epidermis layer would provide new insights into relevant skin disease. Several studies showed that the curvature of rete ridges can vary in ageing or obesity [9]. Other studies found several skin disorders are associated with changes in epidermal junction characterization, such as psoriasis [10]. The proposed method to quantify the curvature relies on measuring the difference between the actual border of the epidermis and a straight reference line connecting the two far sides of the border. The curvature quantification stages can be summarized as follows.

- The border the cornified layers are first extracted from the binary mask of the layer obtained in the previous stage (Figure 4B and 4C). This is simply done by enlarging the mask by one pixel from the top and the bottom followed by subtracting the original mask from it.

- Select the top border (Figure 4D) and connect the far two sides using a theoretical reference line (the red line in Figure 4E).

- Calculate the distances between the points on the actual top border with the reference straight line as explained below.

- The mean of all distances is used a measure to represent the curvature of the epidermis. 
The distance from a line (the reference line) with equation

to the point $(u, v)[11]$ is:

$$
A x+B y+C=0
$$

$$
\text { Distance }=\frac{|A u+B v+C|}{\sqrt{A^{2}+B^{2}}}
$$

The distance has been found between each points on the epidermis curve and the theoretical line (the red line on Figure 4E), which was define by the equation (1). After that, small distances (less that a threshold) are ignored as they are more likely to represent a noise. The mean of distances is calculated for each image to represent the curvature measurement. It is worth mentioning that all images have more or the less the same orientations close to horizontal.

Fig. 4. Representative images for each step of the automated image analysis method to find the curvature in the cornified layer

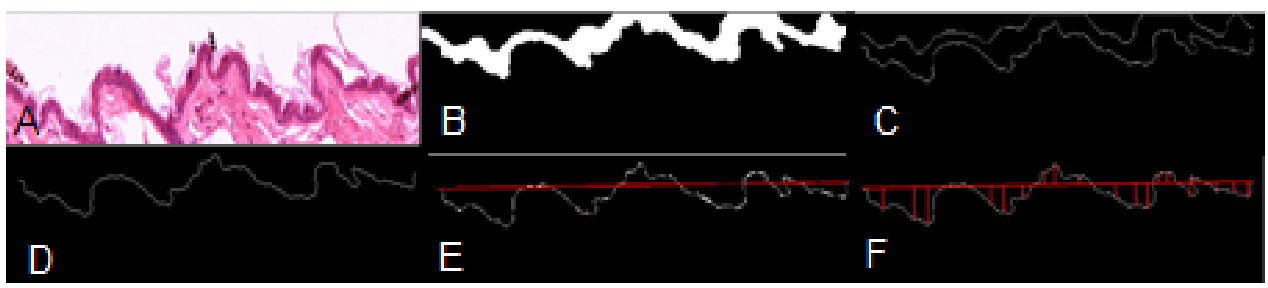

NB: A) Original image. B) Cornified layer. C|) Border for the cornified layer. D) Bottom curvature line. E) Theoretical red line on bottom curvature line. F) Distance between theoretical red line and the bottom curvature line.

\section{Identifying the genes associated with changes in the curvature of the Epidermis.}

This paper follows a stringent protocol described by the MGP to identify interesting genotypes for further analysis [12], which can be summarised as follows. The reference range (RR) method is used to establish the distribution of the WT measurements for each genetic background. If $60 \%$ of the measurements obtained form images of a mutant mice falls outside the $95 \%$ confidence intervals (CI) of the RR range, the knocked-out gene for that mutant line is ladled as an interesting gene i.e. a gene that has led to significant changes to the epidermis curvature. The lower and upper $95 \%$ CI were computed by the following equations:

$$
\text { Lower } 95 \% \text { CI=-S*1.64+M }
$$


Where the $\mathrm{M}$ is the mean of the WT measurements, and $\mathrm{S}$ is the standard deviation. If the number of measurements from a mutant that are below the lower $95 \% \mathrm{CI}$ was $>60 \%$ or above the upper $95 \%$ CI was $>60 \%$, the gene of that particular mutant line is consider for future investigation by biologists.

\section{$4 \quad$ Experiments.}

\subsection{Dataset}

The proposed method is tested on a dataset generated by the Wellcome Trust Sanger Institute (WTSI), which generates mouse genetic and phenotypic data, and distributes this data and resources to the scientific community. The program at WTSI to functionally annotate the mouse genome is illustrated. The primary phenotyping data generated by WTSI aims to discover genes involved in diseases. There are many other research projects that focus on the biological functions of genes in the mouse genetics area by WTSI, such as mouse behaviours, cancer and developmental genetics [13] .Data can be accessed via the mouse resources portal (http://www.sanger.ac.uk/mouseportal/). H\&E stained skin from 16 week-old female mice. The testing data set has 5714 H\&E stained image with $20 \mathrm{X}$ of magnification and dimensions 1444X908. The images contained 29 wild-type (WT) animals and 116-knockout animal selected randomly by WTSI. There were 2-3 slides available from each animal, and 6-10 images per slide (captured at the magnification and resolution above) were created.

\subsection{Results}

As it is not feasible to manually check the segmentation accuracy of 5714 images, we took random sample of 500 images. By close manual examination, we found that 18 images out of the 500 image were not segmented successfully as shown in Figure 5 i.e. the segmentation algorithm achieved an accuracy about $96.4 \%$.

As for the analysis results based on the RR method explained above, we identify 32 genotypes responsible for changes in the Epidermis curvature. Figure 6 shows 18 genotypes (e.g. Actn4/ Actn4) in which the curvature measurements are bigger than the RR 95\% CI upper threshold whereas Figure 7 shows 14 genotypes (e.g. Wdr37) in which the curvature measurements are smaller than the RR 95\% CI lower threshold

Figure 8 shows an example of these interesting genotypes, particularly Actn4/ Actn 4 that increase the curvature in the epidermis layer and $\mathrm{Wdr} 37$ that is associated with a decrease in curvature. Figure 8 shows example of images, which have increased and decrease in the curvature of the epidermis layer of the H\&E microscopic images in the mice skin. 
Fig. 5. Segmentation accuracy, A) Bad segmentation. B) Good segmentation

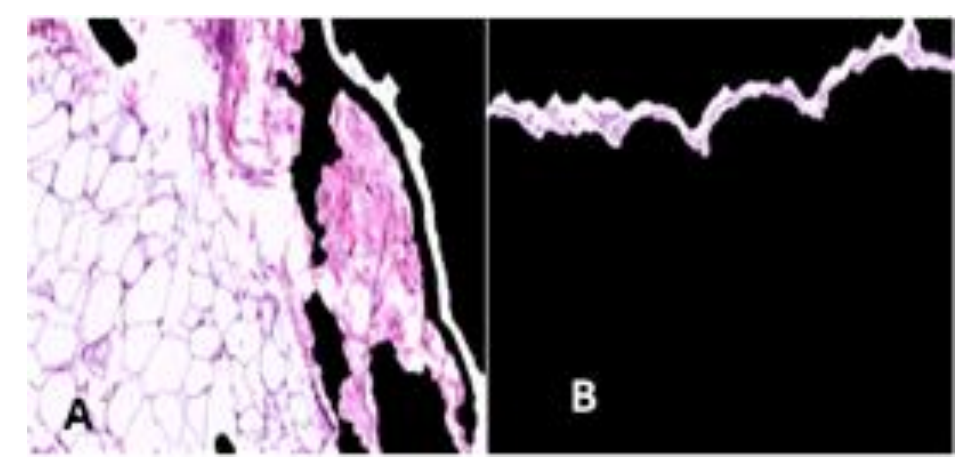

\subsection{Curvature Accuracy Compared With Manual}

Twenty five images was selected randomly from development data set (5714 images), then a manual technique was used to quantify the curvature in the epidermis layer to determine the truth distance values. Next the comparing operation has been done, which was clarified in Figure 7, with the automatic result, which $R^{2}$ value, was 0.90 , has used to measure the approach data to the fitted linear line ground truth value of the distance was found, by calculate the distance between the epidermis layer and the reference line, which was connected between a start point and end point of the epidermis layer as shown in Figure 6, after that, the mean value was calculated for all distances values, for every single image, and then the comparison was applied between the mean value of the manual and the automatic method. 
Fig.6. Manual method for quantifying the curvature of epidermis layer

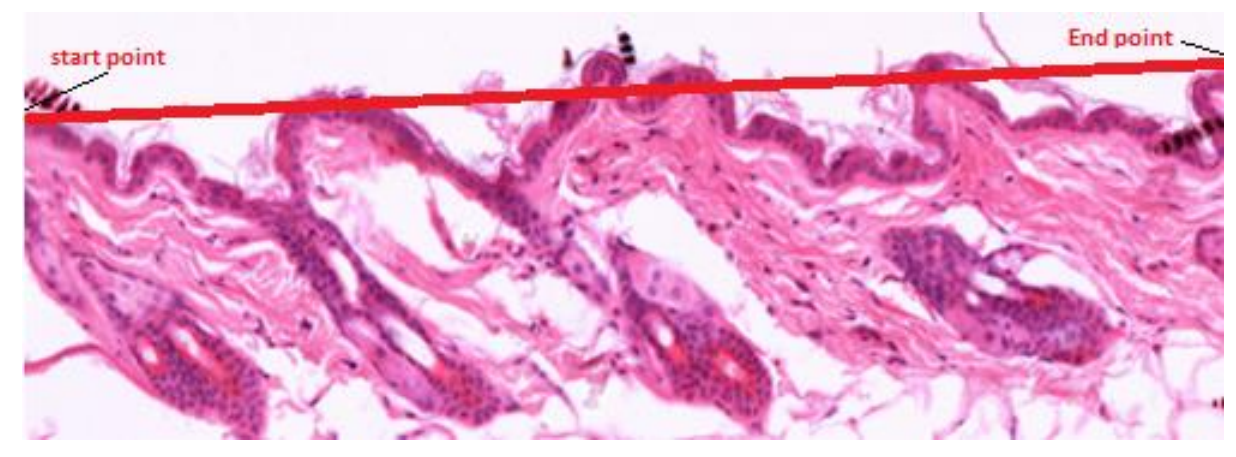

Fig.7. Comparison between manual and automatic method

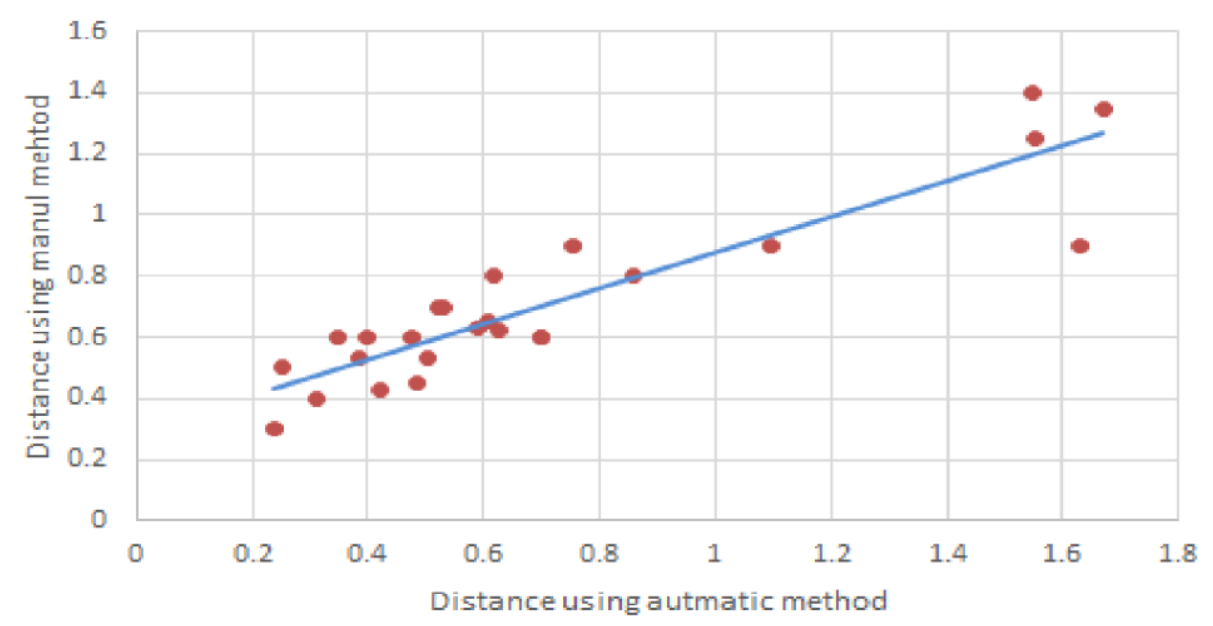




\section{Conclusion}

Biological image processing and analysis provides techniques that help scientists to evaluate the effects of environmental exposure and physiological changes in a research context. These techniques may also be used to evaluate the effect of the treatments and drug efficiency in the context of the drug discovery. All of these analysis techniques could also be applicable clinically. In all applications, novel combinations of image processing/analysis techniques and pipelines will save time and are expected to produce more accurate results that will ultimately help or improve the speed and quality of dermatology and cosmetic treatments. This paper described a method for quantifying the curvature of the epidermis to help identifying genes responsible for changes in the curvature. Experiments on a large set of microscopic images of mutant and WT mice demonstrated the effectiveness of the proposal.

Fig. 8. Curvature in development data. From analysing 5714 development images, 18 genotypes showed curvature measurements bigger than RR 95\% CI upper threshold.

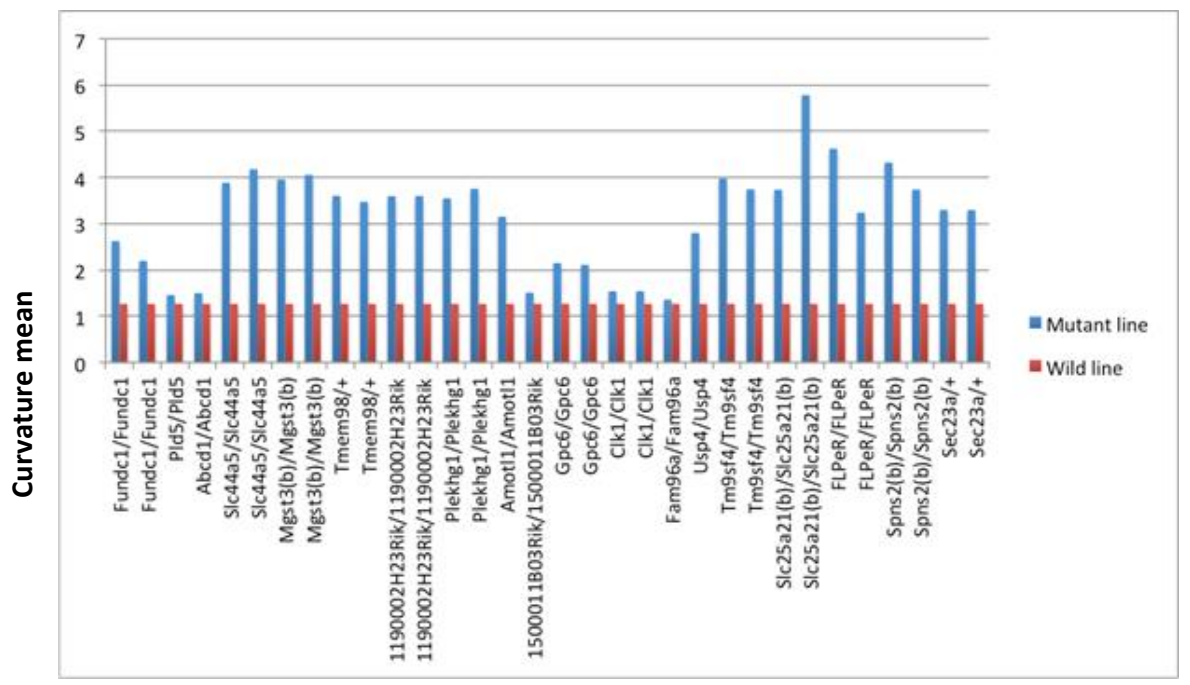


Fig. 9. Curvature in development data. From analysing 5714 development images, 14 genotypes showed curvature measurements smaller than RR 95\% CI lower threshold.

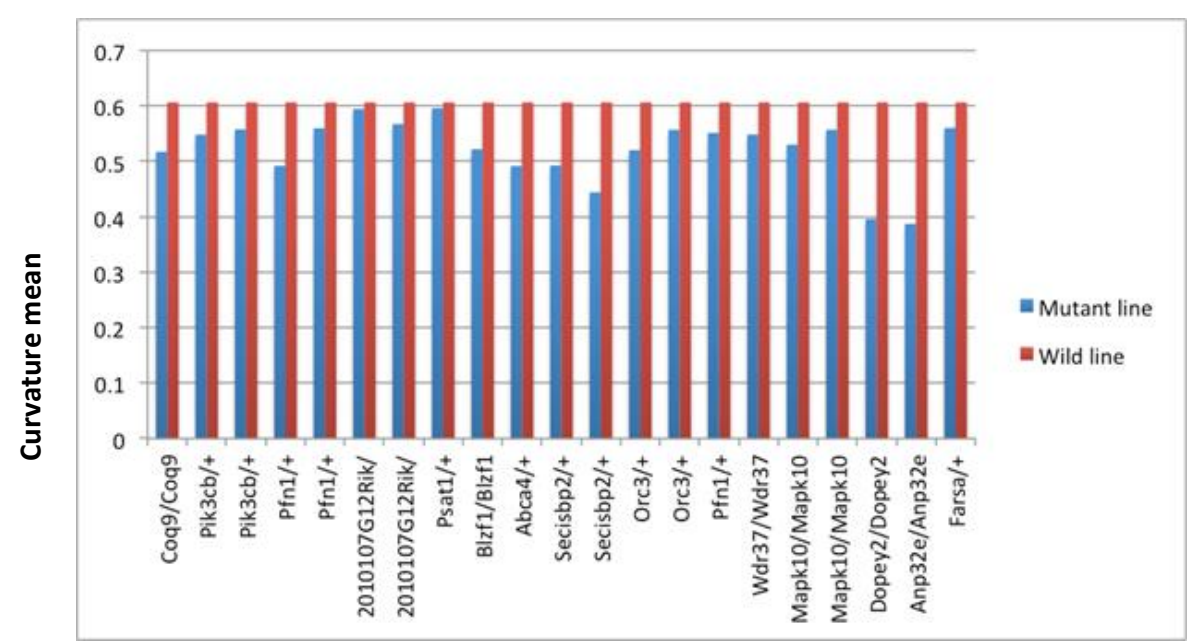

Fig. 10. Automatic estimation of interesting genes for curvature in epidermis layer.

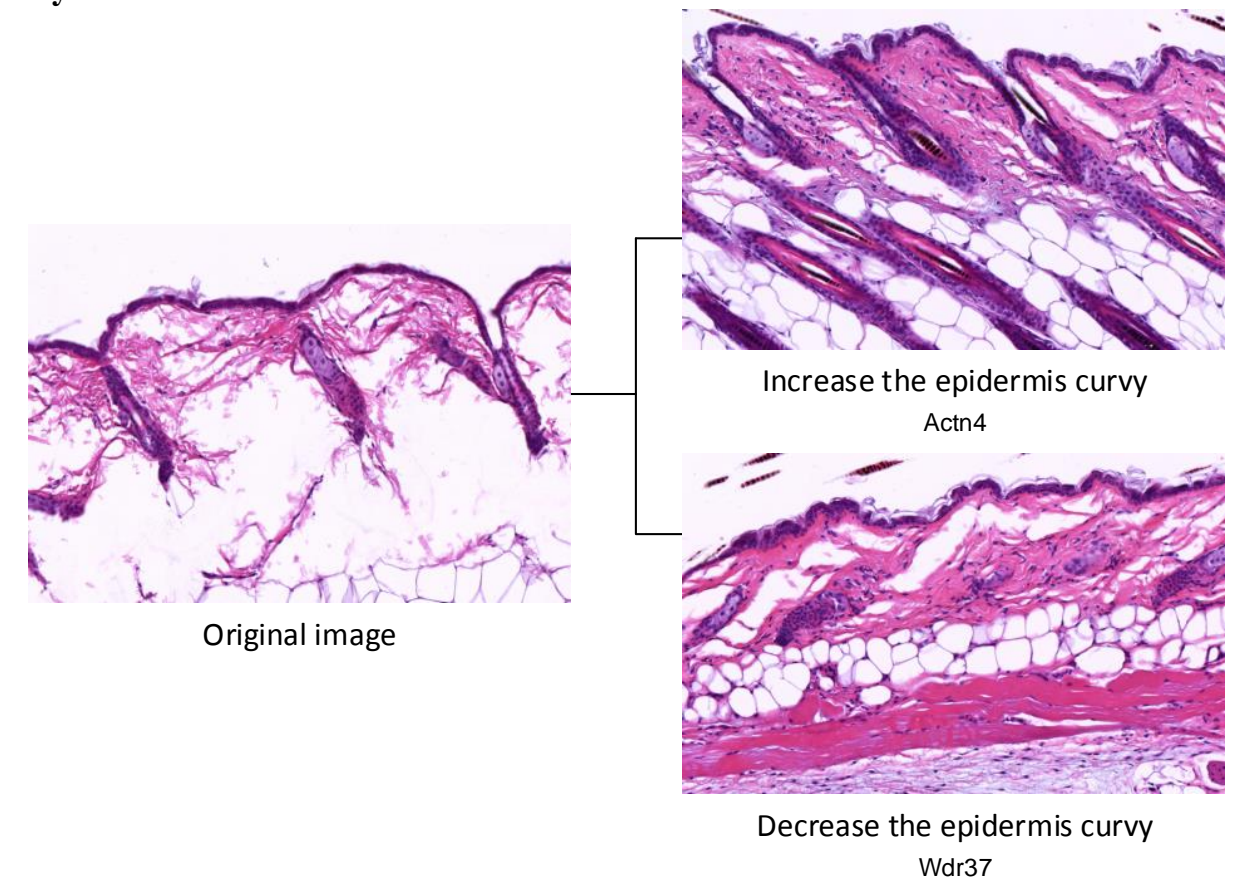




\section{Bibliography}

1. F. S. Collins and E. Jordan are with the National Human Genome Research Institute, National Institutes of Health, Bethesda, MD 20892, USA. A. Patrinos is with the Office of Biological and Environmental Research, Department of Energy, Washington, DC 20585," "New Goals for the U.S. Human Genome Project: 19982003," Science, 23 October 1998.

2. Zaidi, Z. and S. W. Lanigan (2010). Skin: structure and function. Dermatology in Clinical Practice, Springer, Skin: structure and function. Dermatology in Clinical Practice, 2010.

3. S. C. Taylor, "Skin of color: biology, structure, function, and implications for dermatologic disease," Journal of the American Academy of Dermatology, vol., 2002.

4. J. Weissman, T. Hancewicz and P. Kaplan, "Optical coherence tomography of skin for measurement of epidermal thickness by shapelet-based image analysis," Optics Express, vol. 12, no. 23, pp. 5760-5769, 2004

5. M. Ali and B. Hadj, "Segmentation of OCT Skin images by classification of speckle statistical parameters," in Image Processing (ICIP), 2010 17th IEEE International Conference on, 2010.

6. S. Kurugol, J. G. Dy, D. H. Brooks and M. Rajadhyaksha, "Pilot study of semiautomated localization of the dermal/epidermal junction in reflectance confocal microscopy images of skin," Journal of biomedical optics, vol. 16, no. 3, pp. 3, 2011.

7. A. Li, J. Cheng, A. P. Yow, C. Wall, D. W. K. Wong, H. L. Tey and J. Liu, "Epidermal segmentation in high-definition optical coherence tomography," in Engineering in Medicine and Biology Society (EMBC), 2015 37th Annual International Conference of the IEEE, 2015.

8. Saif Hussein,Joanne Selway, Sabah Jassim, Hisham Al-Assam, "Automatic layer segmentation of H\&E microscopic images of mice skin," in SPIE, 2016.

9. K. Honda, T. Sakaguchi, K. Sakai, C. Schmedt, A. Ramirez, J. L. Jorcano, A. Tarakhovsky, H. Kamisoyama and T. Sakai, "Epidermal hyperplasia and papillomatosis in mice with a keratinocyte-restricted deletion of csk," Carcinogenesis, vol. 28, no. 10, pp. 2074-2081, 2007.

10. S. Kurugol, J. G. Dy, M. Rajadhyaksha, K. W. Gossage, J. Weissmann and D. H. Brooks, "Semi-automated algorithm for localization of dermal/epidermal junction in reflectance confocal microscopy images of human skin," in SPIE BiOS, 2011.

11. J. Libby, Teaching Practical Uses for Algebra, Geometry and Trigonometry, McFarland \& Company, 2017.

12. J. H. Duncan Bassett., Apostolos Gogakos, Jacqueline K. White, Holly Evans, Richard M. Jacques,, "Rapid-Throughput Skeletal Phenotyping of 100 Knockout Mice Identifies 9 New Genes That Determine Bone Strength," PLOS genetic, , 2012.

13. K. Liakath-Ali, V. E. Vancollie, E. Heath, D. P. Smedley, J. Estabel, D. Sunter, T. DiTommaso, J. K. White, R. Ramirez-Solis, I. Smyth and others, "Novel skin phenotypes revealed by a genome-wide mouse reverse genetic screen," Nature communications, vol. 5, 2014. 